\title{
The Bed Nucleus of the Stria Terminalis Modulates Learning after Stress in Masculinized But Not Cycling Females
}

\author{
Debbie A. Bangasser and Tracey J. Shors \\ Department of Psychology and Center for Collaborative Neuroscience, Rutgers University, Piscataway, New Jersey 08854
}

\begin{abstract}
Exposure to an acute stressful event enhances classical eyeblink conditioning in male rats, but severely impairs conditioning in female rats. Previous studies have demonstrated that the hippocampus and amygdala critically mediate this effect in both sexes. Thus, although stress affects learning in opposite ways, the structures involved are similar. Previously, we found that the bed nucleus of the stria terminalis (BNST) is also necessary for the enhanced learning after stress in male rats. Here we used BNST inactivation to determine whether the BNST, a sexually dimorphic brain region, is required in female rats for the impaired learning after stress. Interestingly, inactivation of the BNST did not prevent the stress-induced impairment of conditioning in females. Thus, unlike the hippocampus and amygdala, the BNST is critically involved in the modulation of learning by stress in males, but not in females. This exclusive involvement in males may be caused by the sex differences within the BNST. These sex differences result from early testosterone exposure, which masculinizes brain regions including the BNST. Previously, we reported that, like males, females with brains that are masculinized at birth learn better after stressful experience. Here we found that the enhanced learning after stress in masculinized females was prevented by BNST inactivation, just like in males. These data suggest that a masculinized BNST is required for the enhanced learning after a stressful experience. Importantly, together these studies indicate that males and females can engage different brain structures to modulate learning after a stressful experience.
\end{abstract}

Key words: bed nucleus of the stria terminalis; sex difference; stress; classical conditioning; masculinization; testosterone

\section{Introduction}

Stressful life events can affect the acquisition of new information. However, little is known about the structures necessary for the modulation of learning by stress. In our laboratory, we reliably find that exposure to an acute stressful event enhances the acquisition of classical eyeblink conditioning in male rats, whereas it impairs conditioning in female rats (Wood et al., 2001; Hodes and Shors, 2005). This effect has been used to identify the circuitry required for stress to modulate learning. Previously we demonstrated that hippocampal lesions prevent both the enhanced conditioning after stress in males and the impaired conditioning after stress in females, without disrupting learning itself (Bangasser and Shors, 2007). Additionally, inactivation of the basolateral amygdala during stressor exposure prevents the modulation of learning by stress in both sexes (Shors and Mathew, 1998; Waddell et al., 2008). These data suggest that similar brain structures in males and females are engaged by stress to modify learning, despite the fact that the direction of the effect of stress on learning is different in males versus females (i.e., enhanced in males vs impaired in females).

The bed nucleus of the stria terminalis (BNST) is a sexually

Received Feb. 25, 2008; revised May 13, 2008; accepted May 14, 2008.

This work was supported by National Institute of Mental Health Grants 59970 (T.J.S.) and AG19957-06 (D.A.B.), and National Science Foundation Grant IOB-0444364 (T.J.S.). Special thanks to C. Dalla for technical support.

Correspondence should be addressed to Dr. Tracey J. Shors, Department of Psychology, Rutgers University, 152 Frelinghuysen Road, Piscataway, NJ 08854. E-mail: shors@rutgers.edu.

DOI:10.1523/JNEUROSCI.0831-08.2008

Copyright $\odot 2008$ Society for Neuroscience $\quad$ 0270-6474/08/286383-05\$15.00/0 dimorphic brain region (del Abril et al., 1987; Han and De Vries, 2003) that mediates stress and anxiety-related behaviors in male rats (females have not been tested) (Fendt et al., 2003; Walker et al., 2003). Previously, we found that BNST lesions attenuate the stress-induced enhancement of eyeblink conditioning in males. Additional studies using a temporary inactivation technique revealed that the BNST is not required during stressor exposure, but instead is necessary during training for this effect. BNST inactivation did not disrupt the stress response or learning itself, which suggests a specific role in mediating the lasting effects of stress on conditioning, at least in males (Bangasser et al., 2005). Here we wanted to determine whether the BNST mediates impaired learning in females after a stressful experience. If the BNST is required for the stress-induced impairment of learning in females, this would support the idea that the same circuitry mediates both enhancements and impairments of learning after stress. However, because the BNST is sexually dimorphic, it is possible that the BNST is not necessary for the effect in females, which would make the BNST the first brain region identified that modulates stress effects on learning in one sex but not the other.

Early testosterone exposure masculinizes the brain. The organizational effects of early testosterone exposure result in both volumetric and neurochemical sex differences in the BNST (del Abril et al., 1987; Han and De Vries, 2003). Additionally, adult females with masculinized brains respond to an acute stressor like males do, demonstrating an enhancement of conditioning (Shors and Miesegaes, 2002). Because the BNST is necessary for the enhanced learning in males after stress, we hypothesized that it 
would be similarly necessary in females with masculinized brains. To test this hypothesis, female rats were exposed to testosterone early in development, and as adults they were implanted with bilateral cannulas targeting the BNST. The BNST was then inactivated to determine whether it was required to mediate enhanced learning after stress in masculinized females.

\section{Materials and Methods}

Subjects and testosterone treatment. Female Sprague Dawley rats were bred in our colony from Harlan stock. For experiments examining the organizational effect of testosterone, female pups were injected three times subcutaneously with $0.02 \mathrm{ml}$ of $25 \mathrm{mg} / \mathrm{ml}$ ( $0.5 \mathrm{mg}$ total) of testosterone propionate (TP; Sigma-Aldrich) dissolved in sesame seed oil in the afternoon of postnatal day 1 (P1), P3, and P5. This dose has been demonstrated previously to masculinize the BNST (Han and De Vries, 2003). All rats were weaned at P25 and put into same sex group housing arrangements. At P60, rats were removed from their group housing and moved into a single housing arrangement in either wire mesh cages or standard shoe box cages, where they were allowed to acclimate for at least $7 \mathrm{~d}$ before surgery. All rats used in the study were between 67 and $90 \mathrm{~d}$ old. Rats had ad libitum access to food and water and were maintained on a $12 \mathrm{~h}$ light/dark cycle.

Surgeries. Each rat was anesthetized with sodium pentobarbital (40 $\mathrm{mg} / \mathrm{kg}$ females, i.p.) along with atropine $(0.04 \mathrm{mg} / \mathrm{kg}$, i.p. $)$ to reduce salivary secretions. Boosters of each were given as needed. After securing the rat in the stereotaxic instrument, four anchoring screws were secured, and the skull and dura over the BNST were removed. Guide cannulas (23 gauge; Plastics One) were implanted bilaterally, along with stylets (tips flush with guides) aimed at the BNST (anteroposterior, $-0.4 \mathrm{~mm}$ relative to bregma; mediolateral, $\pm 1.4 \mathrm{~mm}$ from midline; dorsoventral, -6.4 $\mathrm{mm}$ from the surface of the brain, angled $15^{\circ}$ toward midline to avoid puncturing the ventricles). The cannulas were secured and a head stage for eyeblink conditioning was fixed to the skull and anchoring screws with dental cement. For eyeblink conditioning, two pairs of electrodes (0.005 inch insulated stainless-steel wire) were attached to a head stage and implanted through the upper eyelid (obicularis occuli muscle). The insulation was removed from a section of each electrode for contact with the muscle.

Vaginal cytology and verification of masculinization. In females, the stress-induced impairment of eyeblink conditioning is affected by estrogen levels, with the largest deficit occurring when females are stressed during late diestrus (low estrogen levels) and then trained during proestrus (high estrogen levels) (Wood and Shors, 1998; Wood et al., 2001). Therefore, we monitored the estrous cycle so that females would be stressed in diestrus and trained in proestrus. To this end, after surgery vaginal cytology was examined daily until it was verified that each subject cycled through all phases of the estrous cycle. Loose vaginal cells were removed with cotton tipped applicators soaked in saline and rolled onto slides. The slides were stained with $1 \%$ toluidine blue and the estrous phase was assessed. Each phase of the estrous cycle was identified using the following characteristics: proestrus is marked by purple staining of epithelial cell nuclei, estrus is marked by dark blue clumped cornified cells, and diestrus is marked by dark leukocytes with scattered epithelial cells. Animals with abnormal cycles were excluded from the study. Successful testosterone treatments and masculinization of female rats was verified in adulthood by inspection of the vagina. In normal females, vaginal canalization occurs during puberty at $\sim$ P35 (Hodes and Shors, 2005). Females that were injected with testosterone never exhibited vaginal canal opening and, thus, were not lavaged.

Conditioning apparatus. The conditioning apparatuses consisted of an inner chamber $(22 \times 26 \times 25 \mathrm{~cm})$ with metal walls and a grounded floor grid within a sound-attenuating outer chamber $(51 \times 52 \times 35 \mathrm{~cm})$. A program written in VIEWDAC controlled a white noise generator attached to a speaker that administered an $80 \mathrm{~dB}$ white noise conditioned stimulus (CS). A shock generator (Lafayette Instrument) administered the $0.65 \mathrm{~mA}$ eyelid shock unconditioned stimulus (US). Each chamber was illuminated with a $7.5 \mathrm{~W}$ bulb. Ten conditioning apparatuses were used simultaneously.
Recording and analysis of eyeblinks. Eyeblinks were detected by changes in eyelid electromyographic (EMG) activity. EMG electrodes were connected to a differential amplifier with a $300-500 \mathrm{~Hz}$ bandpass filter and amplified 10,000 times. EMG signals were digitized at $1 \mathrm{kHz}$ and analyzed with a Visual Basic software program. EMG activity for each trial was compared with a $250 \mathrm{~ms}$ pre-CS baseline. Eyeblinks were defined as changes in EMG activity that exceeded a minimum value of $0.3 \mathrm{mV}$ and the mean pre-CS baseline by at least 4 SDs for a minimum duration of 7 of $10 \mathrm{~ms}$.

Transient BNST inactivation. During the microinfusion procedure, stylets were replaced with infusion cannulas extending $1 \mathrm{~mm}$ beyond the tip of the guide cannula. Infusion cannulas were connected to a microinfuser pump via polyethylene tubes attached to a $10 \mu \mathrm{l}$ Hamilton syringe. The entire system was backloaded with distilled water, with a small air bubble separating the distilled water from the drug or vehicle solution. To temporarily inactivate the BNST, muscimol, a $\mathrm{GABA}_{\mathrm{A}}$ receptor agonist, was bilaterally infused $[0.25 \mu \mathrm{l}$ per side at a rate of $0.1 \mu \mathrm{l} / \mathrm{min} ; 1$ $\mu \mathrm{g} / \mu \mathrm{l}$ in artificial CSF (aCSF)]. The vehicle (aCSF) alone was infused in the untreated animals. After infusion, cannulas were left in place for 1 min to allow for proper diffusion. Concentration and volume were the same as used previously in males (Fendt et al., 2003; Bangasser et al., 2005).

Acclimation and stressor exposure. After at least 1 week of recovery, when females were in the late diestrus phase of their cycle, they were placed in the conditioning chamber for $40 \mathrm{~min}$ for them to acclimate to the conditioning environment. After acclimation, all of the rats used in experiment 1 were infused with either aCSF or muscimol. Rats that were randomly assigned to the unstressed groups were returned to their home cages, whereas those assigned to the stressed groups were exposed to the uncontrollable stressor. Those exposed to the stressor were taken into another room and put in a restraining tube located within a dark, soundattenuating chamber. Electrodes attached to the tail delivered 30 shocks $(1 \mathrm{~s}, 1 \mathrm{~mA})$ at a rate of 1 per minute.

Trace eyeblink conditioning. Twenty-four hours after acclimation and stressor exposure for some, when rats were in the proestrus phase of their cycle, they began training with trace eyeblink conditioning. In the trace eyeblink conditioning procedure, the CS $(250 \mathrm{~ms}, 83 \mathrm{~dB})$ is separated from the periorbital shock US ( $100 \mathrm{~ms}, 0.65 \mathrm{~mA})$ by a $500 \mathrm{~ms}$ trace interval. Trials were presented ( 150 trials/d for $4 \mathrm{~d}$ ) in blocks of 10 in the following order: one CS-alone trial, four paired trials, one US-alone trial, and four paired trials with an intertrial interval of $25 \pm 5$ s. Eyeblinks were defined as conditioned responses (CRs) if they occurred during the $500 \mathrm{~ms}$ trace interval. Before each day of training, all subjects received a BNST infusion of either aCSF or muscimol according to the experimental design detailed below.

Experimental design. The first experiment was designed to determine when, if ever, the BNST was critically involved in the stress-induced impairment of conditioning in females. Five experimental conditions were as follows. (1) An unstressed control group $(n=7)$ received vehicle infusions the day before training (when stressor exposure would have occurred) and before each day of training. (2) A stressed control group $(n=5)$ received vehicle infusion before stressor exposure and before training each day. (3) An experimental group $(n=6)$ received BNST inactivation during stressor exposure. (4) An experimental group $(n=8)$ received a vehicle infusion during stressor and then received BNST inactivation during training. (5) A control group $(n=5)$ to test for performance deficits was left unstressed (and received a vehicle infusion when the stressor would have occurred) but then received BNST inactivation during training.

The second experiment was designed to determine whether the malelike stress effect on conditioning found in adult female rats with masculinized brains required the BNST (Shors and Miesegaes, 2002). Because the BNST was only necessary during training in males, we examined the same time point in masculinized females. Three groups were as follows. (1) An unstressed control group $(n=6)$ that received vehicle infusions before each day of training. (2) A stressed control group $(n=7)$ that received vehicle infusions before each day of training. (3) A stressed experimental group $(n=6)$ that received BNST inactivation before each day of training. 

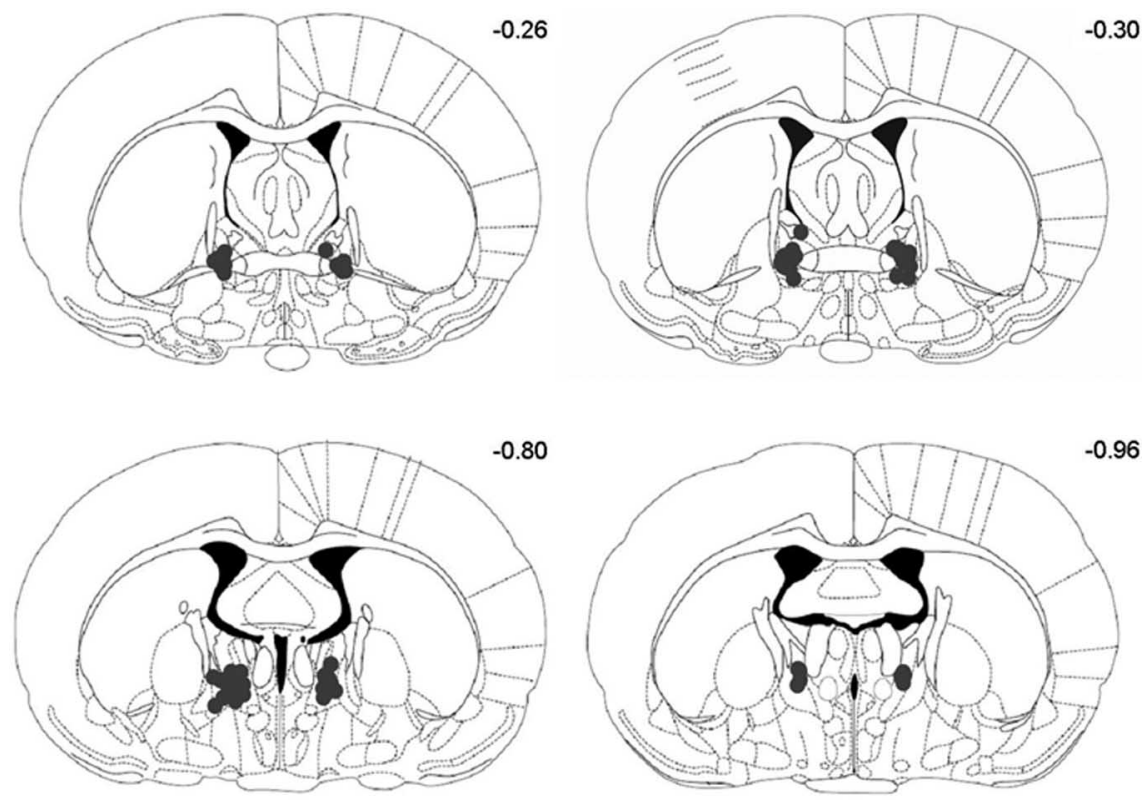

Figure 1. Rats were implanted with bilateral cannulas aimed at the BNST. The cannulas were angled at $15^{\circ}$ to avoid the ventricles. Tip placements from rats included in this study are shown here on coronal sections (Paxinos and Watson, 1997).

\section{A) Vehicle infusions}

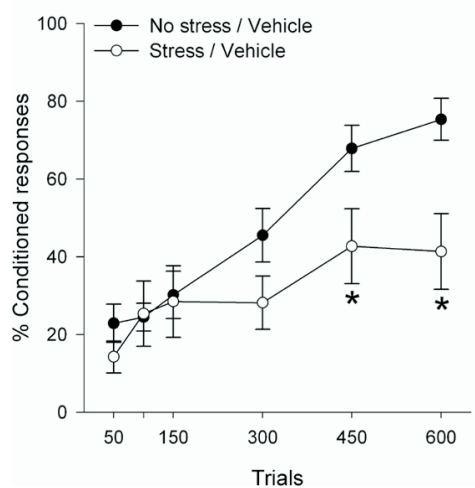

\section{B) BNST inactivation}

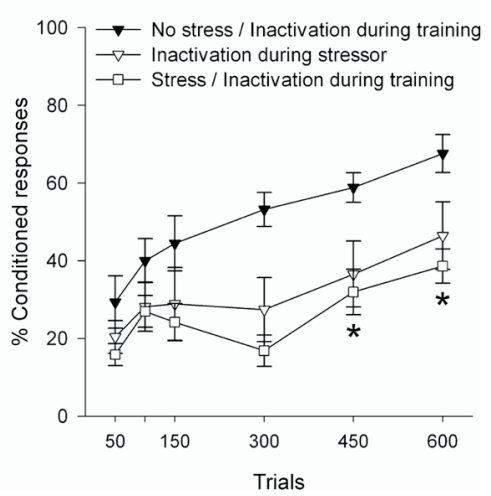

Figure 2. $\quad A$, As expected, stressed vehicle-infused controls $(n=5)$ emitted fewer conditioned responses than unstressed controls $(n=7)$. $B$, BNST inactivation during the stressor $(n=6)$ did not attenuate this impairment. Surprisingly, BNST inactivation after stressor exposure during training $(n=8)$ also failed to prevent the stress-induced impairment of conditioning. Because muscimol infusions during training $(n=5)$ did not prevent conditioned responding itself, these results are not attributable to performance deficits. Thus, unlike males, in females the BNST is not necessary for modulation of conditioning by stress. Data are represented as mean \pm SEM. The percentage of CRs is presented for the 600 training trials. ${ }^{*} p<0.05$ versus unstressed vehicle-infused controls.

Histology. Rats were anesthetized with a sublethal dose of sodium pentobarbital and brains were extracted and postfixed in 10\% formalin for a minimum of $1 \mathrm{~d}$. Sucrose was added to the solution (final concentration of sucrose $30 \%$ ) for an additional $3 \mathrm{~d}$ to prepare tissue for frozen sectioning. Coronal sections $(50 \mu \mathrm{m})$ containing target regions were cut with a cryostat. Every fifth section was mounted onto gelled slides and then stained with cresyl violet so that tip placement could be assessed. A rater, who was unaware of the behavioral data, assessed cannula placements (Fig. 1). Rats were excluded from the study if cannulas were misplaced outside the BNST $(n=3)$ or placed in the ventricle $(n=2)$. The number of subjects per condition listed above reflects the subjects with accurate cannula placements (Fig. 1).

\section{Results}

The BNST in cycling females is not required

One group of rats received muscimol to inactivate of the BNST during stressor exposure, whereas another received BNST inac- tivation after stress during training. Both groups were compared with muscimol and vehicle-infused controls. Using the percentage of CRs across blocks of trials as a measure of learning, a mixed-factors ANOVA revealed that there was an interaction between experimental group and learning $\left(F_{(44,286)}=1.96 ; p<0.05\right)$ (Fig. $2)$. To evaluate when group differences emerged, each day of training (150 trials) was assessed with a one-way ANOVA that compared the average number of CRs for each group. These analyses revealed that the groups differed from each other by day $3\left(F_{(3,22)}=5.40 ; p<0.05\right)$. NewmanKeuls post hoc tests revealed that, as expected, stressor exposure impaired conditioning in vehicle-infused controls compared with their unstressed counterparts $(p<0.05)$. Additionally, rats that received BNST inactivation during the stressor demonstrated impaired conditioning $(p<0.05)$. This result was not surprising because, in male rats, BNST inactivation during the stressor was also ineffective. However, stressed females that received BNST inactivation during training continued to demonstrate impaired conditioning $(p<0.05)$. Further manipulations indicated that this result was not attributable to the performance effects caused by muscimol infusions, because unstressed rats with BNST inactivation during training performed similarly to vehicle-infused controls $\left(F_{(11,110)}=1.76\right.$; $p>0.05)$. Thus, unlike males, the BNST was not required for modulating conditioning by stress in females.

\section{The BNST is essential in masculinized females}

In the second experiment, females with masculinized brains were stressed and then trained on trace eyeblink conditioning either with or without BNST inactivation during training. Previous studies have shown that under certain training conditions, there is a sex difference in learning between males and females, with females acquiring trace eyeblink conditioning faster than males (Wood et al., 1998). Here we wanted to determine whether cycling females learned better than masculinized females. A mixed-factors ANOVA revealed a significant interaction between condition (masculinized vs cycling) and trials $\left(F_{(11,121)}=5.4 ; p<0.05\right)$ and post hoc tests revealed that cycling females emitted more conditioned responses than masculinized females $(p<0.05)$. Thus, like males, masculinized females did not perform as well as cycling females. A mixed-factors ANOVA to determine whether BNST inactivation prevented the stress effect on conditioning in masculinized females revealed that there was an interaction between experimental group and learning $\left(F_{(22,176)}=2.30 ; p<0.05\right)$ (Fig. 3). Group differences emerged by day $3\left(F_{(2,16)}=4.16 ; p<0.05\right)$. Masculinized females that were exposed to the stressor emitted more conditioned re- 


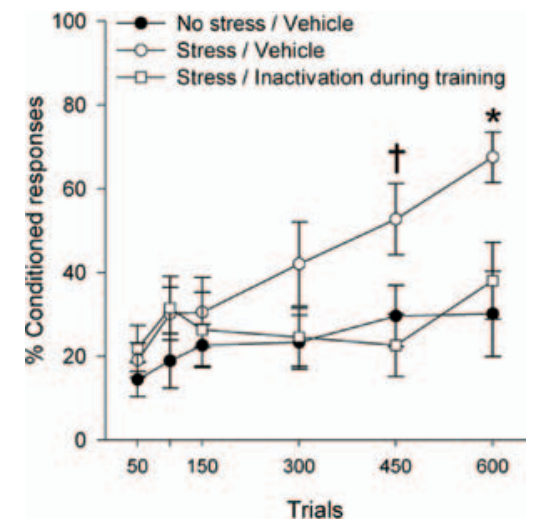

Figure 3. In masculinized females, stressor exposure enhanced conditioning in vehicleinfused controls ( $n=7$ ) compared with their unstressed counterparts $(n=6)$. BNST inactivation $(n=6)$ prevented the enhanced conditioning. Thus, like males, masculinized females demonstrate enhanced conditioning after stress and this effect requires the BNST. Data are represented as mean \pm SEM. The percentage of CRs is presented for the 600 training trials. ${ }^{\dagger} p=0.056$; ${ }^{*} p<0.05$ versus unstressed vehicle-infused controls.

sponses than their unstressed counterparts (day 3, $p=0.056$; day $4, p<0.05)$. This effect was attenuated by BNST inactivation during training (days 3 and $4, p<0.05$ ). Thus, like in males, BNST inactivation prevents the stress-induced enhancement of conditioning in masculinized females.

\section{Discussion}

Previously, we found that the hippocampus and amygdala form part of the critical circuitry for mediating the stress effect on eyeblink conditioning in both male and female rats (Shors and Mathew, 1998; Bangasser and Shors, 2007; Waddell et al., 2008). These findings suggest that although the direction of the effect of stress on learning is different between the sexes (i.e., enhanced in males, whereas impaired in females) the underlying circuitry is the same. In the present study, we wanted to determine whether the BNST, which mediates the stress-induced enhancement of conditioning in males, also mediates the impaired conditioning in females. Neither BNST inactivation during the stressor nor during training prevented the impaired learning after stress in females. Because muscimol infusions during training did not disrupt conditioning itself, these results are not attributable to performance effects. Thus, unlike in males, the BNST is not a critical part of the circuitry for modulating conditioning after stressful experience in female rats. Furthermore, in contrast to the role of the hippocampus and amygdala, the BNST critically mediates the stress effect in one sex but not the other. These results are the first, to our knowledge, to demonstrate that males and females use different brain structures to modulate learning after stressful experience.

Previous studies have determined that females with brains that are masculinized by early testosterone exposure respond like males during adulthood, with stress enhancing rather than impairing their learning (Shors and Miesegaes, 2002). Here we tested whether, like males, females with masculinized brains required the BNST for enhanced learning after stress. As expected, vehicle-infused females with masculinized brains performed like males: under unstressed conditions they did not learn as well as cycling females, but after exposure to the stressor they outperformed unstressed controls. Importantly, BNST inactivation during training prevented the enhanced learning after stress in masculinized females. This result suggests that either the neurochemical or volumetric masculinization of the BNST critically mediates the enhancing effects of stress on eyeblink conditioning. Additionally, it indicates that effects of brain masculinization are not limited to reproductive-related behaviors, as traditionally thought, but can be extended to include effects of stress on associative learning.

\section{Organizational versus activational effects of hormones}

The present findings indicate that males and females use different structures to modulate learning after an acute stressful event. This suggests that the mechanism for enhanced learning is different from that of impaired learning after stress. Our previous findings on the influences of sex hormones also support this idea. It is not simply that circulating testosterone results in enhanced conditioning, whereas circulating estrogen results in impaired conditioning. Instead, the enhanced conditioning is attributable to organizational effects of testosterone, whereas the impaired conditioning is attributable to activational effects of ovarian hormones. As demonstrated here, early testosterone exposure results in the male-like increase in conditioning after the stressful event (Shors and Miesegaes, 2002). This effect occurred without additional testosterone treatment during puberty, which indicates that testosterone exposure early in development is sufficient to establish the masculinized effect. Additionally, castration does not disrupt the enhanced learning after stress (Shors and Miesegaes, 2002), indicating that circulating testosterone levels are not sufficient for the male effect. In females, the impaired conditioning after stress is not attributable to organizational effects of ovarian hormones (Shors and Miesegaes, 2002), but instead is dependent on the presence of relatively high concentrations of ovarian hormones after puberty. Indeed, the effect is most evident when estrogen levels are elevated during proestrus (Wood et al., 2001). Additionally, the detrimental effect of stress on learning can be prevented by either removing the ovaries or treating females with an estrogen receptor antagonist (Wood and Shors, 1998; Wood et al., 2001). Finally, stress does not impair learning in females when they are tested during stages of life when estrogen levels are low (e.g., during the prepubescent, postpartum, and "postmenopausal" stages) (Hodes and Shors, 2005, 2006; Leuner and Shors, 2006). Together these data, when combined with the present study, suggest that there are different mechanisms for enhanced versus impaired learning after stress.

\section{Circuitry mediating impaired conditioning}

As noted previously, the hippocampus and amygdala are required for the stress effect on learning in both males and females (Shors and Mathew, 1998; Bangasser and Shors, 2007; Waddell et al., 2008). Thus, it is possible that the amygdala and hippocampus are sufficient for modulating the impaired conditioning after stress in females. Alternatively, a different brain region could be necessary for the stress effect on conditioning in females. For example, the prefrontal cortex may be necessary in females because it is anatomically positioned to mediate these effects (McLaughlin et al., 2001). Additionally, stress can induce retraction of prefrontal cortex dendrites, which correlates with deficits in learning (Izquierdo et al., 2006). The prefrontal cortex has a relatively high concentration of estrogen receptors (Zhang et al., 2002) and some regions are apparently larger in women than in men (Gur et al., 2002). Thus, the prefrontal cortex may be uniquely involved in mediating impaired learning in females after stress, a possibility that is currently being investigated. 


\section{BNST and sex differences in mental illness}

Stressful life experiences are associated with the emergence of stress-related mental disorders, such as posttraumatic stress disorder and depression. Women are twice as likely to suffer from these disorders as men (Seeman, 1997). Our results indicate that the BNST is necessary to modulate learning after stress in males but not in females. Because learning is enhanced after a stressful experience in males, it could be proposed that the BNST mediates a resistance to the consequences of stressful events. Minimally, the present results suggest the intriguing possibility that men and women use different brain structures to learn after a stressful event. They also underscore the importance of considering sex differences when treating mental illnesses induced by stressful life experience.

\section{References}

Bangasser DA, Shors TJ (2007) The hippocampus is necessary for enhancements and impairments of learning following stress. Nat Neurosci 10:1401-1403.

Bangasser DA, Santollo J, Shors TJ (2005) The bed nucleus of the stria terminalis is critically involved in enhancing associative learning after stressful experience. Behav Neurosci 119:1459-1466.

del Abril A, Segovia S, Guillamon A (1987) The bed nucleus of the stria terminalis in the rat: regional sex differences controlled by gonadal steroids early after birth. Brain Res 429:295-300.

Fendt M, Endres T, Apfelbach R (2003) Temporary inactivation of the bed nucleus of the stria terminalis but not of the amygdala blocks freezing induced by trimethylthiazoline, a component of fox feces. J Neurosci 23:23-28.

Gur RC, Gunning-Dixon F, Bilker WB, Gur RE (2002) Sex differences in temporo-limbic and frontal brain volumes of healthy adults. Cereb Cortex 12:998-1003.

Han TM, De Vries GJ (2003) Organizational effects of testosterone, estradiol, and dihydrotestosterone on vasopressin mRNA expression in the bed nucleus of the stria terminalis. J Neurobiol 54:502-510.
Hodes GE, Shors TJ (2005) Distinctive stress effects on learning during puberty. Horm Behav 48:163-171.

Hodes GE, Shors TJ (2006) Learning during middle age: a resistance to stress? Neurobiol Aging 28:1783-1788.

Izquierdo A, Wellman C, Holmes A (2006) Brief uncontrollable stress causes dendritic retraction in infralimbic cortex and resistance to fear extinction in mice. J Neurosci 26:5733-5738.

Leuner B, Shors TJ (2006) Learning during motherhood: a resistance to stress. Horm Behav 50:38-51.

McLaughlin J, Flaten MA, Chachich M, Powell DA (2001) Medial prefrontal lesions attenuate conditioned reflex facilitation but do not affect prepulse modification of the eyeblink reflex in rabbits. Exp Brain Res 140:318 -325.

Paxinos G, Watson C (1997) The rat brain in stereotaxic coordinates, Ed 3. Orlando, FL: Academic.

Seeman MV (1997) Psychopathology in women and men: focus on female hormones [review]. Am J Psychiatry 154:1641-1647.

Shors TJ, Mathew PR (1998) NMDA receptor antagonism in the lateral/ basolateral but not central nucleus of the amygdala prevents the induction of facilitated learning in response to stress. Learn Mem 5:220-230.

Shors TJ, Miesegaes G (2002) Testosterone in utero and at birth dictates how stressful experience will affect learning in adulthood. Proc Natl Acad Sci USA 99:13955-13960.

Waddell J, Bangasser DA, Shors TJ (2008) The basolateral nucleus of the amygdala is necessary to induce the opposing effects of stressful experience on learning in males and females. J Neurosci 28:5290-5294.

Walker DL, Toufexis DJ, Davis M (2003) Role of the bed nucleus of the stria terminalis versus the amygdala in fear, stress, and anxiety [review]. Eur J Pharmacol 463:199-216.

Wood GE, Shors TJ (1998) Stress facilitates classical conditioning in males, but impairs classical conditioning in females through activational effects of ovarian hormones. Proc Natl Acad Sci USA 95:4066-4071.

Wood GE, Beylin AV, Shors TJ (2001) The contribution of adrenal and reproductive hormones to the opposing effects of stress on trace conditioning in males versus females. Behav Neurosci 115:175-187.

Zhang JQ, Cai WQ, Zhou DS, Su BY (2002) Distribution and differences of estrogen receptor beta immunoreactivity in the brain of adult male and female rats. Brain Res 935:73-80. 